\title{
Brunneroma presenting with radiological features of duodeno-duodenal intussusception
}

\author{
W.Y.M. Abeysekera', MBBS, W.D.D. de Silva, MBBS MD MRCS², P. Pragatheswaran, \\ MBBS $^{1}$, K.M.K. Somaratne MBBS, MD, MRCS $^{2}$, A.S.K. Banagala, MBBS MS FRCSI ${ }^{3}$ \\ 1 Registrar in Surgery, Colombo South Teaching Hospital, Kalubowila, Sri Lanka. \\ 2 Senior Registrar in Surgery, Colombo South Teaching Hospital, Kalubowila, Sri Lanka. \\ 3 Consultant Surgeon, Colombo South Teaching Hospital, Kalubowila, Sri Lanka
}

Key words: Brunneroma; Duodeno-duodenal

Intussusception.

\section{Introduction}

Benign tumours of the duodenum are exceedingly rare (incidence $<0.01$ ). Approximately $11 \%$ of these are Brunner's gland adenoma/Brunneroma or polypoidal hamartoma which is a benign, proliferative lesion of the submucosal Brunner's glands [1]. Duodenoduodenal intussusception (DDI) is a rare presentation of such a tumour with only a few cases reported thus far. We present a case of a large Brunneroma in the first part of the duodenum(D1), with radiological features of DDI. However the clinical symptom profile and intra-operative findings were not suggestive of acute or chronic duodenal obstruction.

\section{Case presentation}

A 67 year old lady presented with a history of melaena of 1 week duration with a vague, intermittent epigastric pain with regurgitation for more than a year. Examination was unremarkable except for the marked pallor. Iron deficiency anaemia was confirmed with haemoglobin $6.6 \mathrm{~g} / \mathrm{dl}$ and serum ferritin $21 \mu \mathrm{g} / \mathrm{L}$.

Abdominal ultrasonography revealed a $4.3 \mathrm{~cm} \times 3.2$ $\mathrm{cm} \times 4.4 \mathrm{~cm}$ hyperechoic mass in left hypochondrial region and CECT of the abdomen suggested a short segment of duodenum appeared intussuscepted into the proximal portion of the third part of duodenum (D3) (Figure 1). The distal part of D3 appeared intussuscepted into the D4 segment. No definite lead point demonstrated and no significant proximal or

Correspondence: W.Y.M Abeysekera, No. 10A, Mission Lane, Pita-Kotte, Kotte,

Email: yohan.abeysekera@yahoo.com

The Sri Lanka Journal of Surgery 2012; 30(1):36-38. distal bowel distension seen to suggest obstruction.

In Upper gastrointestinal endoscopy a mobile polypoidal mass was identified on the posterosuperior wall of D1 (Figure 2). Histology of the endoscopic punch biopsy was inconclusive. Polypectomy was performed through a gastrotomy incision by mobilizing the pedunculated polyp (Figure 3) in to the stomach from the posterior wall of D1. Histopathology confirmed a Bruneroma with a diameter of $6 \mathrm{~cm}$. Neither during endoscopy nor surgery was there evidence of DDI.

\section{Discussion}

Duodeno-duodenal or duodeno-jejunal intussusception is an extremely rare manifestation with only a handful of reported cases [2]. Enteroenteric intussusception itself is a rare event in adults and this is always secondary to the presence of a lead point like a hamartoma, Meckels diverticulum or a tumour. Cruveilhier described one of the earliest cases of Brunner gland adenomas co-incidentally associated with fatal duodenal intussusception [3].

DDI is exceptionally rare because the retroperitoneal situation fixes the duodenal wall. The radiological diagnosis of duodenal intussusception is based on characteristic features seen in contrast upper GI series, CT and ultrasound. The classic 'coiled spring sign' usually apparent in contrast films of jejunal intussusception, is not so easily evident in the duodenum. CT or ultrasound evidence of duodenal intussusception is based on the appearance of the 'target sign' due to the multi-layered appearance of the telescoping bowel walls [4]. 


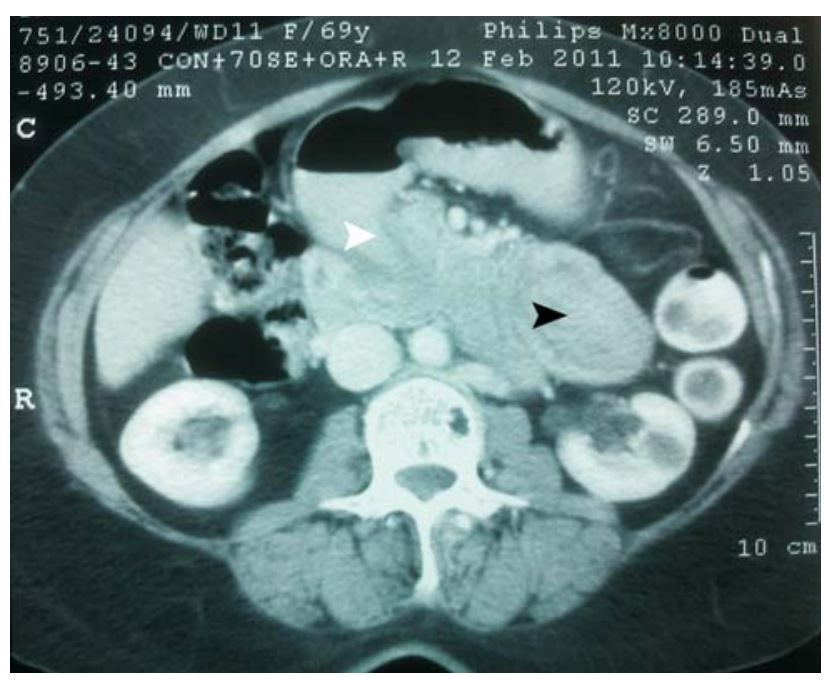

Figure 1.CT image demonstrating a large mass (black arrowhead) seen prolapsed in the third part of the duodenum, along with the intussusception of second part of the duodenum in to the third part (white arrowhead)

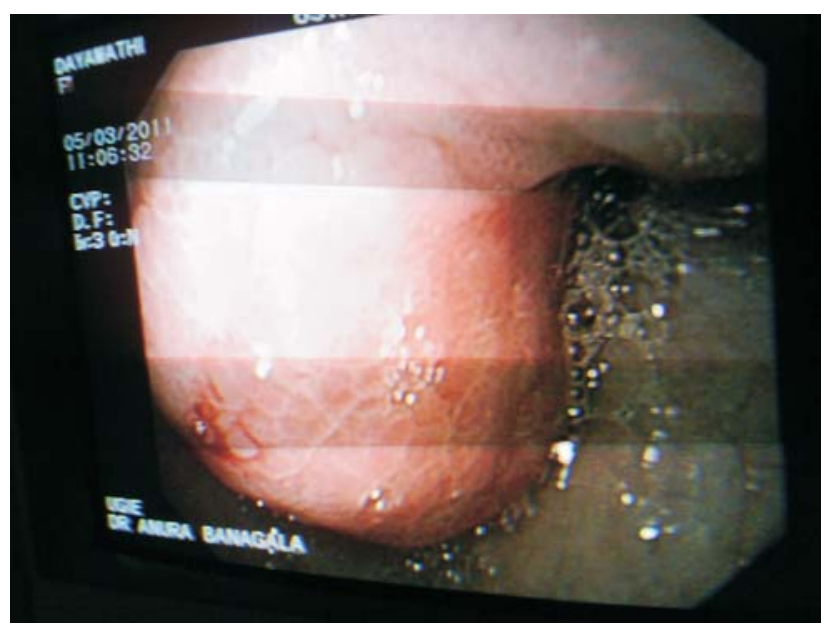

Figure 2. Endoscopic image showing a large polypoidal lesion in the first part of the duodenum

In our case the CT was suggestive of DDI in the proximal and distal parts of the duodenum, but she had no features to suggest either acute or chronic duodenal obstruction. Despite the retroperitoneal fixation, as a result of mucosal elongation or slipping, duodenal tumours can migrate even up to the jejunum [5]. Saida et al while reviewing a series of duodenal tumours makes the interesting submission that some cases of duodenal intussusception based radiological signs may not be due to a true intussusception but a radiological illusion due to mucosal prolapse as a result of mucosal elongation or slipping [6]. It is

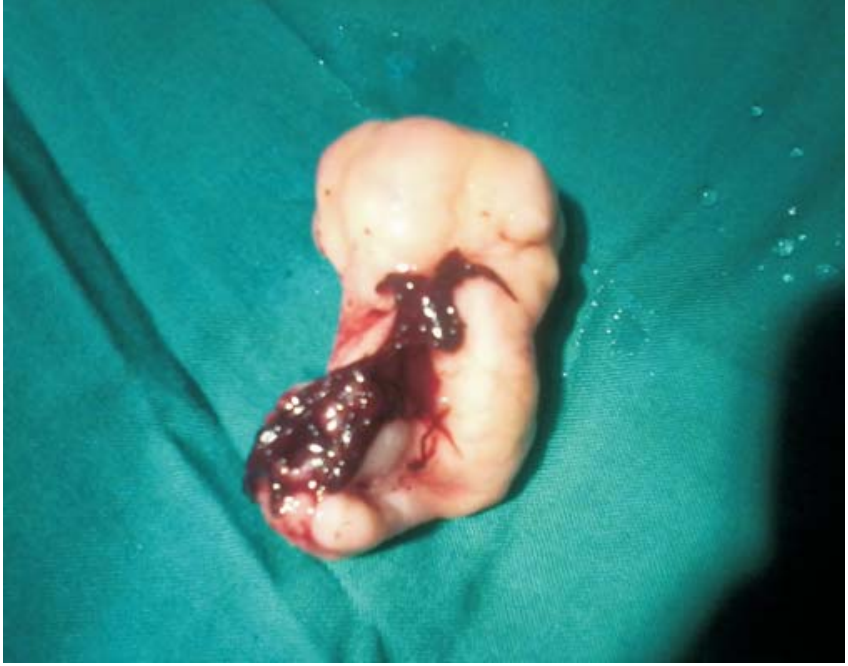

Figure 3. Polyp after removal via a gastrotomy incision

possible that this was the case in our patient as well. Alternatively it may be that there was spontaneous resolution of a true duodenal intussusception.

\section{Acknowledgements}

Dr. D.D. Ranasinghe and Dr. W.A. Epa, consultant radiologists at the Colombo South Teaching Hospital for the imaging support in the management of this patient.

\section{References}

1. Botsford TW, Crowe P, Croker DW. Tumors of the small intestine: A review of experience with 115 cases including a report of a rare case of malignant hemangioendothelioma. Am J Surg 1962; 103:358-65

2. Petersen JM, Felger TS, Goldstein JD. Gastroduodenal intussusception secondary to a giant Brunner gland hamartoma. Gastroenterol Hepatol. 2008;4:471-473

3. Cruveilhier J. Anatomie Pathologique du Corps humain. Paris. JB Balliere; 1835.

4. Madanur MA, Mula VR, Patel D, Rathinaswamy A, Madanur AA. Periampullary carcinoma presenting as duodenojejunal intussusception: a diagnostic and therapeutic dilemma. Hepatobiliary Pancreat Dis Int 2008; 7: 658-660

5. Taams J, Huizinga WK, Somers SR. The wandering ampulla-duodenal-jejunal 
intussusception of a carcinoid tumour with displacement of the bile duct to the left iliac fossa. A case report. S Afr J Surg 1992; 30: 153155

\section{Key Learning Points}

6. Saida Y, Matsueda K, Itai Y. Distal migration of duodenal tumors: simple prolapse or Intussusception? Abdom Imaging 2002;27:9-14

- Adult intussusception including duodeno-duodenal intussusception is a rare event which is always secondary to the presence of a lead point like a tumor or diverticulum.

- Duodenal intussusception based radiological signs may not be due to a true intussusception but a radiological illusion due to mucosal prolapse. 